\title{
Zero Measure Spectrum for the Almost Mathieu Operator
}

\author{
Y. Last \\ Department of Physics, Technion - Israel Institute of Technology, 32000 Haifa, Israel
}

Received: 13 September 1993

\begin{abstract}
We study the almost Mathieu operator: $\left(H_{\alpha, \lambda, \theta} u\right)(n)=u(n+1)+u(n-$ $1)+\lambda \cos (2 \pi \alpha n+\theta) u(n)$, on $l^{2}(Z)$, and show that for all $\lambda, \theta$, and (Lebesgue) a.e. $\alpha$, the Lebesgue measure of its spectrum is precisely $|4-2| \lambda||$. In particular, for $|\lambda|=2$ the spectrum is a zero measure cantor set. Moreover, for a large set of irrational $\alpha$ 's (and $|\lambda|=2$ ) we show that the Hausdorff dimension of the spectrum is smaller than or equal to $1 / 2$.
\end{abstract}

\section{Introduction}

In this paper, we study the almost Mathieu (also called Harper's) operator on $l^{2}(Z)$. This is the (bounded, self adjoint) operator $H_{\alpha, \lambda, \theta}$, defined by:

$$
\begin{gathered}
H_{\alpha, \lambda, \theta}=H_{0}+V_{\alpha, \lambda, \theta}, \quad\left(H_{0} u\right)(n)=u(n+1)+u(n-1), \\
\left(V_{\alpha, \lambda, \theta} u\right)(n)=\lambda \cos (2 \pi \alpha n+\theta) u(n),
\end{gathered}
$$

where $\alpha, \lambda, \theta \in R$.

$H_{\alpha, \lambda, \theta}$ is a tight binding model for the Hamiltonian of an electron in a one dimensional lattice, subject to a commensurate (if $\alpha$ is rational) or incommensurate (if $\alpha$ is irrational) potential. It is also related to the Hamiltonian of an electron in a two dimensional lattice, subject to a perpendicular magnetic field $[11,13]$ (in which case the relevant energy spectrum is the union over $\theta$ of the energy spectra of $H_{\alpha, \lambda, \theta}$ ).

The almost Mathieu operator has been studied by many authors [1-13, 15, 17-24, 26], and many of its spectral characteristics are known. Our main result in this paper is:

Theorem 1. If $\alpha$ is an irrational, for which there is a sequence of rationals $\left\{p_{n} / q_{n}\right\}$ obeying:

$$
\lim _{n \rightarrow \infty} q_{n}^{2}\left|\alpha-\frac{p_{n}}{q_{n}}\right|=0
$$

Work partially supported by the GIF 
then for every $\lambda, \theta \in R$ :

$$
|\sigma(\alpha, \lambda, \theta)|=|4-2| \lambda||
$$

where $\sigma(\alpha, \lambda, \theta)$ is the spectrum of $H_{\alpha, \lambda, \theta}$, and $|\cdot|$ denotes Lebesgue measure.

Remarks. 1) The set of irrationals characterized in Theorem 1 is precisely the set of irrationals having unbounded continued fraction expansions. This set is known to have full Lebesgue measure [16].

2) The $\theta$ independence part of Theorem 1 is immediate, since, for irrational $\alpha$, $\sigma(\alpha, \lambda, \theta)$ itself is known to be independent of $\theta[9]$.

The equality $|\sigma(\alpha, \lambda, \theta)|=|4-2| \lambda||$ was conjectured by Aubry and Andre [1] to hold for every irrational $\alpha$. It was later studied by Thouless [21], and by Avron, van Mouche, and Simon [3], who established the inequality $|\sigma(\alpha, \lambda, \theta)| \geq|4-2| \lambda||$ (for every $\alpha, \lambda, \theta)$.

For $|\lambda| \neq 2$, Theorem 1 has already been proved in [17]. The main theme of the current paper is the handling of the case $|\lambda|=2$, for which we prove:

Lemma 1. Let $p, q \in N$ be relatively prime, and denote:

$$
S(\alpha, \lambda) \equiv \bigcup_{\theta} \sigma(\alpha, \lambda, \theta) ;
$$

then:

$$
\frac{2(\sqrt{5}+1)}{q}<|S(p / q, 2)|<\frac{8 e}{q}
$$

(where $e \equiv \exp (1)=2.71 \ldots$ ).

It should be remarked that a similar (though somewhat weaker) lower bound on $|S(p / q, 2)|$ was already established in [18]. It is the upper bound in Lemma 1, which is the main new result of the current paper and from which the completion of the proof of Theorem 1 follows.

Lemma 1 is strongly related to a conjecture of Thouless [21-24], which says:

$$
\lim _{q \rightarrow \infty} q|S(p / q, 2)|=\text { const }=9.32 \ldots
$$

This conjecture was found numerically for sequences with $p, q$ relatively prime and $q \rightarrow \infty$. It was derived analytically only for some sequences with fixed $p$ and $q \rightarrow \infty$ $[22,23,26]$. Some (nonrigorous) analytical argumentation that it should also hold for more general cases was given in [18].

It is interesting to note that since $\sigma(\alpha, \lambda, \theta)$ has no isolated points [9], the vanishing of its measure for $|\lambda|=2$ also implies:

Corollary 1.1. For irrational $\alpha$ as in Theorem $1, \sigma(\alpha, 2, \theta)$ is a (zero measure) Cantor set (i.e. a closed, nowhere dense set, with no isolated points).

Moreover, if $\alpha$ is an irrational which is very well approximated by rationals, we will show that Lemma 1 implies an upper bound on the Hausdorff dimension of $\sigma(\alpha, 2, \theta)$, namely:

Theorem 2. If $\alpha$ is an irrational obeying:

$$
q_{n}^{4}\left|\alpha-\frac{p_{n}}{q_{n}}\right|<C
$$


for some constant $C$, and a sequence of rationals $\left\{p_{n} / q_{n}\right\}$ with $q_{n} \rightarrow \infty$, then:

$$
\operatorname{dim}_{H}(\sigma(\alpha, 2, \theta)) \leq \frac{1}{2}
$$

where $\operatorname{dim}_{H}(\cdot)$ denotes Hausdorf dimension.

Remark. The set of irrationals characterized in Theorem 2 has zero Lebesgue measure, but it contains a dense $G_{\delta}$ set, which makes it "generic" in the commonly used topological sense.

The analysis leading to our results is based on previous findings of Avron, van Mouche, and Simon [3], and this paper is, to a large extent, a continuation of their work. While some parts of this analysis were already carried out in [17] and in [18], for the reader's convenience, we shall repeat the relevant derivations of those papers.

In Sect. 2 we describe some preliminaries and previously obtained results. In Sect. 3 we prove Lemma 1, and in Sect. 4 we prove Theorem 1. Finally, in Sect. 5, we prove Theorem 2.

\section{Preliminaries}

We begin this section with a remark about the considered ranges of $\alpha, \lambda, \theta$. Since $H_{\alpha, \lambda, \theta}$ is invariant under: $\alpha \rightarrow \alpha \pm 1, \theta \rightarrow \theta \pm 2 \pi$, we may always assume: $\alpha \in[0,1]$, $\theta \in[0,2 \pi]$. This has no effect on the correctness of our results for more general values of $\alpha$ and $\theta$. Moreover, a sign change of $\lambda(\lambda \rightarrow-\lambda)$ is equivalent to a translation of $\theta$ by $\pi$. Thus, any quantity or result which is independent of $\theta$ must be invariant under a sign change of $\lambda$, and throughout the rest of the paper we will usually assume: $\lambda \geq 0$.

In what follows we will be largely concerned with the spectral analysis of the almost Mathieu operator at rational frequencies. That is, we will consider $H_{\alpha, \lambda, \theta}$, where $\alpha=p / q, p, q \in N$, and we assume throughout that $p$ and $q$ are relatively prime (i.e. they have no common divisor other than 1). In this case $\sigma(\alpha, \lambda, \theta)$ does depend on $\theta$, and we will also be interested in the two spectral sets:

$$
\begin{aligned}
S(\alpha, \lambda) & \equiv \bigcup_{\theta} \sigma(\alpha, \lambda, \theta), \\
S_{-}(\alpha, \lambda) & \equiv \bigcap_{\theta} \sigma(\alpha, \lambda, \theta) .
\end{aligned}
$$

These sets are also well define for irrational $\alpha$, but in this case: $S_{-}(\alpha, \lambda)=S(\alpha, \lambda)=$ $\sigma(\alpha, \lambda, \theta)$. As we shall see later, the set $S(\alpha, \lambda)$ has good continuity properties (in $\alpha$ ), and our results for irrational $\alpha$ are essentially based on the study of $S(\alpha, \lambda)$ for rational $\alpha$.

A central role in the spectral analysis of $H_{p / q, \lambda, \theta}$ is played by the discriminant $D_{p / q, \lambda, \theta}(E)$, defined by:

$$
\begin{aligned}
D_{p / q, \lambda, \theta}(E) \equiv & \operatorname{Trace}\left[\left(\begin{array}{cc}
E-V(1) & -1 \\
1 & 0
\end{array}\right)\left(\begin{array}{cc}
E-V(2) & -1 \\
1 & 0
\end{array}\right) \ldots\right. \\
& \left.\left(\begin{array}{cc}
E-V(q) & -1 \\
1 & 0
\end{array}\right)\right],
\end{aligned}
$$

where $V(n) \equiv \lambda \cos (2 \pi(p / q) n+\theta)$. $D_{p / q, \lambda, \theta}(E)$ is a polynomial of order $q$ (in $E$ ) having the following properties (see e.g. [25]): 
(i) $D_{p / q, \lambda, \theta}(E)$ has $q$ real simple zeroes.

(ii) $D_{p / q, \lambda, \theta}(E)$ is larger than or equal to 2 at all its maxima points, and it is smaller than or equal to -2 at all its minima points.

The spectrum $\sigma(p / q, \lambda, \theta)$ is precisely the inverse image under $D_{p / q, \lambda, \theta}(E)$ of the interval $[-2,2]$ (i.e. it is precisely the set of $E$ 's for which: $-2 \leq D_{p / q, \lambda, \theta}(E) \leq 2$ ). Thus, from the properties of $D_{p / q, \lambda, \theta}(E)$ it is seen that $\sigma(p / q, \lambda, \theta)$ is made of $q$ bands (closed intervals), such that $D_{p / q, \lambda, \theta}(E)$ is strongly monotone on each band. A remarkable formula, originally due to Chambers [6] (also see [5] for a proof), gives the $\theta$ dependence of $D_{p / q, \lambda, \theta}(E)$ :

Proposition 2.1. If $p, q$ are relatively prime, then:

$$
D_{p / q, \lambda, \theta}(E)=\Delta_{p / q, \lambda}(E)-2\left(\frac{\lambda}{2}\right)^{q} \cos \theta q,
$$

where $\Delta_{p / q, \lambda}(E) \equiv D_{p / q, \lambda, \pi / 29}(E)$.

Proposition 2.1 implies that $S(p / q, \lambda)$ is precisely the inverse image under $\Delta_{p / q, \lambda}(E)$ of the interval $\left[-2-2(\lambda / 2)^{q}, 2+2(\lambda / 2)^{q}\right]$. Moreover, it shows that if $\lambda>2$ then $S_{-}(p / q, \lambda)=\emptyset$, and if $\lambda \leq 2$ then $S_{-}(p / q, \lambda)$ is the inverse image under $\Delta_{p / q, \lambda}(E)$ of the interval $\left[-2+2(\bar{\lambda} / 2)^{q}, 2-2(\lambda / 2)^{q}\right]$. We remark that from the fact that the above properties (i) and (ii) of $D_{p / q, \lambda, \theta}(E)$ hold for every $\theta$, and from Proposition 2.1, it follows that $\Delta_{p / q, \lambda}(E)$ is larger than or equal to $2+2(\lambda / 2)^{q}$ at all its maxima points, and it is smaller than or equal to $-2-2(\lambda / 2)^{q}$ at all its minima points. Moreover, each of the sets $S(p / q, \lambda)$ and $S_{-}(p / q, \lambda)$ (when it is not empty) is made of $q$ bands, such that $\Delta_{p / q, \lambda}(E)$ is strongly monotone on each band.

An important property of $H_{\alpha, \lambda, \theta}$ is the Aubry duality [1], which allows relating eigenfunctions and spectra of $H_{\alpha, \lambda, \theta}^{\alpha, \lambda}$ to those of $H_{\alpha, 4 / \lambda, \theta}$. The following version of this duality was rigorously proven by Avron and Simon [2]:

Proposition 2.2. For every real $\alpha$ :

$$
S(\alpha, \lambda)=\frac{\lambda}{2} S(\alpha, 4 / \lambda)
$$

Thus, it is sufficient to study $S(\alpha, \lambda)$ for $0 \leq \lambda \leq 2$, since, for $\lambda>2, S(\alpha, \lambda)$ is obtained by Proposition 2.2. from the $\lambda<2$ case.

Avron, van Mouche, and Simon [3] proved the following:

Proposition 2.3. For $0 \leq \lambda \leq 2$ and $p, q$ relatively prime:

$$
\begin{gathered}
\left|S_{-}(p / q, \lambda)\right|=4-2 \lambda . \\
4-2 \lambda \leq|S(p / q, \lambda)| \leq 4-2 \lambda+4 \pi\left(\frac{\lambda}{2}\right)^{q / 2} .
\end{gathered}
$$

In particular, Proposition 2.3 shows that if $0 \leq \lambda<2$, and if $p_{n} / q_{n} \rightarrow \alpha$, where the $p_{n} / q_{n}$ 's are rationals, and $\alpha$ is irrational, then $\left|S\left(p_{n} / q_{n}, \lambda\right)\right| \rightarrow 4-2 \lambda$. If $\lambda=2$ then statement (ii) of Proposition 2.3 becomes useless; but, it was shown in [18] that, in this case, the remarkable exact equality for $\left|S_{-}(p / q, \lambda)\right|$ (statement (i)) translates to an exact equality involving the slopes of $\Delta_{p / q, \lambda}(E)$ at its zero crossings. Namely: 
Proposition 2.4. If $p, q$ are relatively prime, then:

$$
\sum_{\nu=1}^{q} \frac{1}{\left|\Delta_{p / q, 2}^{\prime}\left(E_{\nu}\right)\right|}=\frac{1}{q}
$$

where $\Delta_{p / q, \lambda}^{\prime}(E) \equiv \frac{d}{d E} \Delta_{p / q, \lambda}(E)$, and $E_{1}, E_{2}, \ldots, E_{q}$ are the $q$ zeroes of $\Delta_{p / q, 2}(E)$. Proof. Since $S_{-}(p / q, \lambda)$ is the inverse image under $\Delta_{p / q, \lambda}(E)$ of the interval $\left[-2+2(\lambda / 2)^{q}, 2-2(\lambda / 2)^{q}\right]$, and since $\Delta_{p / q, \lambda}(E)$ is also a polynomial in $\lambda$, we have, for $\lambda<2$, in the limit $\lambda \rightarrow 2$ :

$$
\left|S_{-}(p / q, \lambda)\right| \sim \sum_{\nu=1}^{q} \frac{4-4(\lambda / 2)^{q}}{\left|\Delta_{p / q, \lambda}^{\prime}\left(E_{\nu}\right)\right|} .
$$

Thus, from statement (i) of Proposition 2.3 we obtain:

$$
\sum_{\nu=1}^{q} \frac{1}{\left|\Delta_{p / q, 2}^{\prime}\left(E_{\nu}\right)\right|}=\lim _{\lambda \nearrow 2} \frac{\left|S_{-}(p / q, \lambda)\right|}{4-4(\lambda / 2)^{q}}=\lim _{\lambda \rightarrow 2} \frac{4-2 \lambda}{4-4(\lambda / 2)^{q}}=\frac{1}{q} .
$$

Proposition 2.4 is in the heart of Lemma 1 that we prove in the next section.

For every $\alpha, \lambda \in R$, the set $S(\alpha, \lambda)$ is compact, and, therefore, it has definite edges: $\max S(\alpha, \lambda), \min S(\alpha, \lambda) \in S(\alpha, \lambda)$. The complement of $S(\alpha, \lambda)$ in the interval [ $\min S(\alpha, \lambda), \max S(\alpha, \lambda)]$ is open, and it is therefore a union of countably many (finite) open intervals. We shall refer to such open intervals, when they are chosen to have maximal length, as gaps in $S(\alpha, \lambda)$, and we shall denote their union by $G(\alpha, \lambda)$. That is:

$$
G(\alpha, \lambda) \equiv[\min S(\alpha, \lambda), \max S(\alpha, \lambda)] \backslash S(\alpha, \lambda),
$$

and so we have:

$$
|S(\alpha, \lambda)|=\max S(\alpha, \lambda)-\min S(\alpha, \lambda)-|G(\alpha, \lambda)| .
$$

When $\alpha$ is rational $(\alpha=p / q)$ we have seen that $S(\alpha, \lambda)$ is made of $q$ bands. Thus, it has at most $q-1$ gaps. When $\alpha$ is irrational $S(\alpha, \lambda)$ may have an infinite number of gaps.

We conclude this section by quoting another result of Avron, van Mouche, and Simon [3], this time regarding the continuity properties of $S(\alpha, \lambda)$ :

Proposition 2.5. For every $\lambda>0$, there is a constant $C$, such that if $\left|\alpha-\alpha^{\prime}\right|<C$ (for any $\alpha, \alpha^{\prime} \in R$ ), then for every $E \in S(\alpha, \lambda)$, there is $E^{\prime} \in S\left(\alpha^{\prime}, \lambda\right)$ with:

$$
\left|E-E^{\prime}\right|<6\left(\lambda\left|\alpha-\alpha^{\prime}\right|\right)^{1 / 2} \text {. }
$$

Proposition 2.5 has the immediate corollary:

Corollary 2.1. (i) If $\left|\alpha-\alpha^{\prime}\right|<C$, then for every gap in $S(\alpha, \lambda)$ with midpoint $E_{g}$, and measure $|g|$ larger than $12\left(\lambda\left|\alpha-\alpha^{\prime}\right|\right)^{1 / 2}$, there is a corresponding (containing $\left.E_{g}\right)$ gap in $S\left(\alpha^{\prime}, \lambda\right)$ with measure larger than: $|g|-12\left(\lambda\left|\alpha-\alpha^{\prime}\right|\right)^{1 / 2}$.

(ii) The same continuity as in (i) also holds for the extreme edges of $S(\alpha, \lambda)$, namely, for $\left|\alpha-\alpha^{\prime}\right|<C$ :

$$
\left|\min _{\min } S(\alpha, \lambda)-\max _{\min } S\left(\alpha^{\prime}, \lambda\right)\right|<6\left(\lambda\left|\alpha-\alpha^{\prime}\right|\right)^{1 / 2}
$$




\section{Proof of Lemma 1}

In this section we consider $\lambda=2$ and a fixed rational $p / q$, where $p$ and $q$ are relatively prime. For simplicity of notation we denote: $\Delta(E)$ for $\Delta_{p / q, 2}(E)$ and $S$ for $S(p / q, 2)$. (i) Proof of the upper bound. Consider a nonextermal band of $S: I_{\nu}=\left[E_{1}^{\nu}, E_{2}^{\nu}\right]$, and suppose that $\Delta(E)$ is increasing on $I_{\nu}$. Denote by $E_{\nu}$ the zero of $\Delta(E)$ inside $I_{\nu}$, and by $E_{0}^{\nu}$ the maximum of $\Delta(E)$ just above $E_{2}^{\nu}$. (In principle, we can have $E_{0}^{\nu}=E_{2}^{\nu}$, but typically $E_{0}^{\nu}>E_{2}^{\nu}$, and $E_{0}^{\nu}$ is inside the gap just above $I_{\nu}$ ). Define:

$$
f(E) \equiv \frac{d}{d E}(\ln (\Delta(E))) .
$$

Since $\Delta(E)$ can be expressed as:

$$
\Delta(E)=\prod_{j=1}^{q}\left(E-E_{\jmath}\right)
$$

$f(E)$ can be written as:

$$
f(E)=\sum_{j=1}^{q} \frac{1}{E-E_{j}}
$$

and we have:

$$
f^{\prime}(E) \equiv \frac{d}{d E} f(E)=-\sum_{j=1}^{q} \frac{1}{\left(E-E_{\jmath}\right)^{2}} .
$$

From (3.4) we see that:

$$
f^{\prime}(E)<\frac{-1}{\left(E-E_{\nu}\right)^{2}},
$$

and since $E_{0}^{\nu}$ is a zero of $f(E)$, we have for every $E \in\left(E_{\nu}, E_{0}^{\nu}\right)$ :

$$
f(E)=-\int_{E}^{E_{0}^{\nu}} f^{\prime}\left(E^{\prime}\right) d E^{\prime}>\int_{E}^{E_{0}^{\nu}} \frac{d E^{\prime}}{\left(E^{\prime}-E_{\nu}\right)^{2}}=\frac{1}{E-E_{\nu}}-\frac{1}{E_{0}^{\nu}-E_{\nu}} .
$$

Now, consider $E \in\left(E_{\nu}, E_{2}^{\nu}\right)$. Since $\Delta\left(E_{2}^{\nu}\right)=4$, we have:

$$
\ln \frac{4}{\Delta(E)}=\ln \Delta\left(E_{2}^{\nu}\right)-\ln \Delta(E)=\int_{E}^{E_{2}^{\nu}} f\left(E^{\prime}\right) d E^{\prime}
$$

and by using (3.7) and the estimate (3.6), we obtain:

$$
\ln \frac{4}{\Delta(E)}>\ln \left(\frac{E_{2}^{\nu}-E_{\nu}}{E-E_{\nu}}\right)-1 \text {. }
$$

Equation (3.8) implies:

$$
\frac{4}{\Delta(E)}>\frac{1}{e}\left(\frac{E_{2}^{\nu}-E_{\nu}}{E-E_{\nu}}\right),
$$

which can also be written as:

$$
E_{2}^{\nu}-E_{\nu}<4 e \frac{E-E^{\nu}}{\Delta(E)} .
$$


Since $\Delta\left(E_{\nu}\right)=0$, we obtain from (3.10), by letting $E \rightarrow E_{\nu}$ :

$$
E_{2}^{\nu}-E_{\nu}<\frac{4 e}{\Delta^{\prime}\left(E_{\nu}\right)} .
$$

Clearly, a similar calculation can also be carried for the lower part of the band, by integrating $f^{\prime}(E)$ from the minimum of $\Delta(E)$ just below (or at) $E_{1}^{\nu}$. Thus, we also have:

$$
E_{\nu}-E_{1}^{\nu}<\frac{4 e}{\Delta^{\prime}\left(E_{\nu}\right)}
$$

which (together with (3.11)) implies:

$$
\left|I_{\nu}\right|=E_{2}^{\nu}-E_{1}^{\nu}<\frac{8 e}{\left|\Delta^{\prime}\left(E_{\nu}\right)\right|} .
$$

It's easy to see that (3.13) could be obtained with a similar calculation, also if $\Delta(E)$ was decreasing on $I_{\nu}$. Thus, (3.13) clearly holds for all of the nonextermal bands. If $I_{\nu}$ is an extermal band, we can still make a similar calculation to (3.1)-(3.11) for the "less extermal" part of this band, and obtain either (3.11) (for the lowest band) or (3.12) (for the highest band). But, since $\left|\Delta^{\prime}(E)\right|$ is monotone on an extermal band, the "more extermal" part of such a band must be smaller than its "less extermal" part. Thus, (3.13) holds for every band, and from Proposition 2.4 we obtain:

$$
|S|=\sum_{\nu=1}^{q}\left|I_{\nu}\right|<\frac{8 e}{q} .
$$

(ii) Proof of the lower bound. For each band of $S: I_{\nu}=\left[E_{1}^{\nu}, E_{2}^{\nu}\right]$, we denote the two parts of the band by:

$$
b_{1}^{\nu} \equiv\left[E_{1}^{\nu}, E_{\nu}\right], \quad b_{2}^{\nu} \equiv\left[E_{\nu}, E_{2}^{\nu}\right] .
$$

Since $\Delta^{\prime}(E)$ is a polynomial of order $q-1$, which has $q-1$ distinct real zeroes, $\left|\Delta^{\prime}(E)\right|$ has a single maximum between every two consecutive zeroes of $\Delta^{\prime}(E)$, and it is monotone above and below the extreme zeroes of $\Delta^{\prime}(E)$. Thus, $\left|\Delta^{\prime}(E)\right|$ has a single maximum on each band (which may be at the edge of the band), and it is monotone on every subinterval of the band which does not contain this maximum. This implies that for each band, either for all $E \in b_{1}^{\nu}$ (if the maximum is on $b_{2}^{\nu}$ ), or for all $E \in b_{2}^{\nu}$ (if the maximum is on $b_{1}^{\nu}$ ), we have:

$$
\left|\Delta^{\prime}(E)\right| \leq\left|\Delta^{\prime}\left(E_{\nu}\right)\right|
$$

Since:

$$
\int_{E_{1}^{\nu}}^{E_{\nu}}\left|\Delta^{\prime}(E)\right| d E=\left|\Delta\left(E_{\nu}\right)-\Delta\left(E_{1}^{\nu}\right)\right|=4
$$

and also

$$
\int_{E_{\nu}}^{E_{2}^{\nu}}\left|\Delta^{\prime}(E)\right| d E=\left|\Delta\left(E_{2}^{\nu}\right)-\Delta\left(E^{\nu}\right)\right|=4
$$


we have, either for $i=1$, or for $i=2$ :

$$
\left|b_{\imath}^{\nu}\right|>\frac{4}{\left|\Delta^{\prime}\left(E_{\nu}\right)\right|} \equiv l_{\nu} .
$$

In [18], (3.19) has been used to obtain: $|S|>4 / q$. We shall now improve this bound.

Suppose that $I_{\nu}$ is a nonextermal band and that $\Delta(E)$ is increasing on $I_{\nu}$, and consider the polynomial:

$$
G(E) \equiv \Delta(E)+4,
$$

which has a zero at $E_{1}^{\nu}$. A similar estimate to (3.1)-(3.6) shows that for every $E \in\left(E_{1}^{\nu}, E_{0}^{\nu}\right)$ :

$$
\frac{G^{\prime}(E)}{G(E)}=\frac{d}{d E} \ln G(E)>\frac{1}{E-E_{1}^{\nu}}-\frac{1}{E_{0}^{\nu}-E_{1}^{\nu}} .
$$

By taking $E=E_{\nu}$ in (3.21), we obtain:

$$
\frac{\Delta^{\prime}\left(E_{\nu}\right)}{4}=\frac{G^{\prime}\left(E_{\nu}\right)}{G\left(E_{\nu}\right)}>\frac{1}{\left|b_{1}^{\nu}\right|}-\frac{1}{\left|b_{1}^{\nu}\right|+\left|b_{2}^{\nu}\right|}=\frac{\left|b_{2}^{\nu}\right|}{\left|b_{1}^{\nu}\right|\left(\left|b_{1}^{\nu}\right|+\left|b_{2}^{\nu}\right|\right)},
$$

which implies:

$$
\left|b_{1}^{\nu}\right|>\frac{l_{\nu}\left|b_{2}^{\nu}\right|}{\left|b_{1}^{\nu}\right|+\left|b_{2}^{\nu}\right|} .
$$

Clearly, by considering: $H(E) \equiv \Delta(E)-4$ instead of $G(E)$, and integrating from the minimum of $H(E)$ just below $E_{1}^{\nu}$, we can similarly obtain (3.23) with $b_{1}^{\nu}$ and $b_{2}^{\nu}$ interchanged, namely:

$$
\left|b_{2}^{\nu}\right|>\frac{l_{\nu}\left|b_{1}^{\nu}\right|}{\left|b_{1}^{\nu}\right|+\left|b_{2}^{\nu}\right|}
$$

We have seen that either $\left|b_{1}^{\nu}\right|>l_{\nu}$ or $\left|b_{2}^{\nu}\right|>l_{\nu}$. Suppose that $\left|b_{2}^{\nu}\right|>l_{\nu}$, then (3.23) implies:

$$
\left|b_{1}^{\nu}\right|>\frac{l_{\nu}^{2}}{\left|b_{1}^{\nu}\right|+l_{\nu}}
$$

which can be rewritten as:

$$
\left|b_{1}^{\nu}\right|^{2}+\left|b_{1}^{\nu}\right| l_{\nu}-l_{\nu}^{2}>0 \text {. }
$$

Solving the appropriate quadratic equation shows that (3.26) implies:

$$
\left|b_{1}^{\nu}\right|>\frac{\sqrt{5}-1}{2} l_{\nu}
$$

Similarly, if $\left|b_{1}^{\nu}\right|>l_{\nu}$, (3.24) would imply:

$$
\left|b_{2}^{\nu}\right|>\frac{\sqrt{5}-1}{2} l_{\nu}
$$

so in either case we have:

$$
\left|I_{\nu}\right|=\left|b_{1}^{\nu}\right|+\left|b_{2}^{\nu}\right|>\frac{\sqrt{5}+1}{2} l_{\nu}
$$

and it is clear that (3.29) holds for every nonextermal band. For the case of an extermal band, only one of the inequalities (3.23) or (3.24) can be obtained. But, in this case, 
due to the monotonicity of $\left|\Delta^{\prime}(E)\right|$, we also know which one of the $\left|b_{i}^{\nu}\right|(i=1,2)$ is larger and obeys: $\left|b_{i}^{\nu}\right|>l_{\nu}$. It is easy to verify that $\left|b_{2}^{\nu}\right|>l_{\nu}$ corresponds to the case where (3.23) holds, and that the other case corresponds to (3.24). Thus, we obtain (3.29) for all the bands, and from Proposition 2.4 we get:

$$
|S|>\frac{2(\sqrt{5}+1)}{q} \text {. }
$$

\section{Proof of Theorem 1}

Lemma 4.1. For every $\lambda \in R$, and a sequence of rationals $\left\{p_{n} / q_{n}\right\}$, with $p_{n}, q_{n}$ relatively prime, and $q_{n} \rightarrow \infty$ :

$$
\lim _{n \rightarrow \infty}\left|S\left(p_{n} / q_{n}, \lambda\right)\right|=|4-2| \lambda|| .
$$

Proof. Combining statement (ii) of Proposition 2.3 and Lemma 1 we obtain for every $0 \leq \lambda \leq 2:\left|S\left(p_{n} / q_{n}, \lambda\right)\right| \rightarrow 4-2 \lambda$. From that and from Proposition 2.2 the lemma follows.

Proposition 4.1 (Thouless [21]; Avron, van Mouche, and Simon [3]). For any irrational $\alpha$, and $\lambda, \theta \in R$ :

$$
|\sigma(\alpha, \lambda, \theta)| \geq|4-2| \lambda||
$$

Proof. Let $\left\{g_{j}(\alpha, \lambda)\right\}_{j=1}^{\infty}$ be the gaps in $S(\alpha, \lambda)$ (ordered somehow), and pick some $\varepsilon>0$. Since $\sum_{j=1}^{\infty}\left|g_{\jmath}(\alpha, \lambda)\right|=|G(\alpha, \lambda)|$, there is a finite $J_{\varepsilon}$ such that $\sum_{j=1}^{J_{\varepsilon}}\left|g_{\jmath}(\alpha, \lambda)\right|>$ $|G(\alpha, \lambda)|-\varepsilon$. Now, consider a sequence of rationals: $p_{n} / q_{n} \rightarrow \alpha$. From statement (i) of Corollary 2.1 we have:

$$
\liminf _{n \rightarrow \infty}\left|G\left(p_{n} / q_{n}, \lambda\right)\right| \geq \sum_{j=1}^{J_{\varepsilon}}\left|g_{j}(\alpha, \lambda)\right|>|G(\alpha, \lambda)|-\varepsilon
$$

and from statement (ii):

$$
\lim _{n \rightarrow \infty} \max _{\min } S\left(p_{n} / q_{n}, \lambda\right)=\max _{\min }^{\max } S(\alpha, \lambda)
$$

Thus, from (2.6) we obtain:

$$
|S(\alpha, \lambda)|>\limsup _{n \rightarrow \infty}\left|S\left(p_{n} / q_{n}, \lambda\right)\right|-\varepsilon,
$$

which by Lemma 4.1 implies:

$$
|S(\alpha, \lambda)|>|4-2| \lambda||-\varepsilon .
$$

Since $S(\alpha, \lambda)=\sigma(\alpha, \lambda, \theta)$ and since $\varepsilon$ is arbitrary this completes the proof.

Proof of Theorem 1. Let $\alpha$ be an appropriate irrational, and let $\left\{p_{n} / q_{n}\right\}$ be a sequence of rationals obeying: $\lim _{n \rightarrow \infty} q_{n}{ }^{2}\left|\alpha-p_{n} / q_{n}\right|=0$. Obviously, $q_{n} \rightarrow \infty$, and we can assume $p_{n}, q_{n}$ to be relatively prime. Since there are at most $q_{n}-1$ gaps in $S\left(p_{n} / q_{n}, \lambda\right)$, we obtain from statement (i) of Corollary 2.1 (for $\left|\alpha-p_{n} / q_{n}\right|<C$ ):

$$
|G(\alpha, \lambda)|>\left|G\left(p_{n} / q_{n}, \lambda\right)\right|-12\left(q_{n}-1\right)\left(\lambda\left|\alpha-p_{n} / q_{n}\right|\right)^{1 / 2} .
$$


By (2.6) and statement (ii) of Corollary 2.1 this implies:

$$
|S(\alpha, \lambda)|<\left|S\left(p_{n} / q_{n}, \lambda\right)\right|+12 q_{n}\left(\lambda\left|\alpha-p_{n} / q_{n}\right|\right)^{1 / 2} .
$$

As $n \rightarrow \infty$, we have from Lemma 4.1: $\left|S\left(p_{n} / q_{n}, \lambda\right)\right| \rightarrow|4-2| \lambda||$, and by our assumption on $\left\{p_{n} / q_{n}\right\}: q_{n}\left|\alpha-p_{n} / q_{n}\right|^{1 / 2} \rightarrow 0$. Thus, (4.6) implies:

$$
|\sigma(\alpha, \lambda, \theta)|=|S(\alpha, \lambda)| \leq|4-2| \lambda||
$$

which together with Proposition 4.1 completes the proof.

\section{Proof of Theorem 2}

Lemma 5.1. Let $S \subset R$, and suppose that $S$ has a sequence of covers: $\left\{S_{n}\right\}_{n=1}^{\infty}$, $S \subset S_{n}$, such that each $S_{n}$ is a union of $q_{n}$ intervals, $q_{n} \rightarrow \infty$ as $n \rightarrow \infty$, and for each $n$ :

$$
\left|S_{n}\right|<\frac{C}{q_{n}^{\beta}},
$$

where $\beta$ and $C$ are positive constants; then:

$$
\operatorname{dim}_{H}(S) \leq \frac{1}{1+\beta}
$$

Proof. Let $S_{n}=\bigcup_{\nu=1}^{q_{n}} b_{\nu}^{n}$, where each $b_{\nu}^{n}$ is an interval. Without loss, we can assume that the $b_{\nu}^{n}$ 's are disjoint. Let $t=1 /(1+\beta)$, then:

$$
\frac{1}{q_{n}} \sum_{\nu=1}^{q_{n}}\left|b_{\nu}^{n}\right|^{t} \leq\left(\frac{1}{q_{n}} \sum_{\nu=1}^{q_{n}}\left|b_{\nu}^{n}\right|\right)^{t}
$$

which implies:

$$
\begin{aligned}
\sum_{\nu=1}^{q_{n}}\left|b_{\nu}^{n}\right|^{t} & \leq q_{n}{ }^{1-t}\left(\sum_{\nu=1}^{q_{n}}\left|b_{\nu}^{n}\right|\right)^{t}=q_{n}{ }^{1-t}\left|S_{n}\right|^{t} \\
& \leq q_{n}{ }^{1-t}\left(\frac{C}{q_{n}{ }^{\beta}}\right)^{t}=q_{n}{ }^{1-(1+\beta) t} C^{t}=C^{t}
\end{aligned}
$$

Recall that the Hausdorff dimension of $S$ is given by (see e.g. [14]):

$$
\operatorname{dim}_{H}(S)=\inf \left\{\left.t \in R^{+}\left|\lim _{\delta \rightarrow 0} \inf _{\delta-\text { covers }} \sum_{\nu}\right| b_{\nu}\right|^{t}<\infty\right\},
$$

where a $\delta$-cover is a cover of $S: S \subset \bigcup_{\nu=1}^{\infty} b_{\nu}$, such that each $b_{\nu}$ is an interval, and $\left|b_{\nu}\right|<\delta$. Thus, since $q_{n} \rightarrow \infty$ as $n \rightarrow \infty,(5.2)$ implies: $\operatorname{dim}_{H}(S) \leq 1 /(1+\beta)$.

Proof of Theorem 2. Let $\alpha$ be an appropriate irrational, and let $\left\{p_{n} / q_{n}\right\}$ be a sequence of rationals obeying: $q_{n} \rightarrow \infty$ as $n \rightarrow \infty$, and $q_{n}{ }^{4}\left|\alpha-p_{n} / q_{n}\right|<C$. Clearly, we can 
assume that $p_{n}$ and $q_{n}$ are relatively prime. For each $n, S\left(p_{n} / q_{n}, 2\right)$ is made of $q_{n}$ bands:

$$
S\left(p_{n} / q_{n}, 2\right)=\bigcup_{\nu=1}^{q_{n}}\left[E_{1}^{\nu, n}, E_{2}^{\nu, n}\right]
$$

and by Proposition 2.5, we have (for sufficiently large $n$ ):

$$
S(\alpha, 2) \subset \bigcup_{\nu=1}^{q_{n}}\left[E_{1}^{\nu, n}-6\left(2\left|\alpha-p_{n} / q_{n}\right|\right)^{1 / 2}, E_{2}^{\nu, n}+6\left(2\left|\alpha-p_{n} / q_{n}\right|\right)^{1 / 2}\right] \equiv S_{n}
$$

$S_{n}$ is a cover of $S(\alpha, 2)$ by $q_{n}$ intervals, and from Lemma 1 and our assumptions on $\left\{p_{n} / q_{n}\right\}$ we have:

$$
\begin{aligned}
\left|S_{n}\right| \leq & \left|S\left(p_{n} / q_{n}, 2\right)\right|+12 q_{n}\left(2\left|\alpha-p_{n} / q_{n}\right|\right)^{1 / 2} \\
& <\frac{8 e}{q_{n}}+12 q_{n}\left(\frac{2 C}{q_{n}{ }^{4}}\right)^{1 / 2}=\frac{8 e+12(2 C)^{1 / 2}}{q_{n}} .
\end{aligned}
$$

Thus, Lemma 5.1 implies: $\operatorname{dim}_{H}(S(\alpha, 2)) \leq 1 / 2$.

Acknowledgements. It is a pleasure to thank J. Avron, B. Simon, and M. Wilkinson for useful discussions.

\section{References}

1. Aubry, S., Andre, G.: Analyticity breaking and Anderson localization in incommensurate lattices. Ann. Israel Phys. Soc. 3, 133-164 (1980)

2. Avron, J., Simon, B.: Almost periodic Schrödinger operators. II. The integrated density of states. Duke Math. J. 50, 369-391 (1983)

3. Avron, J., van Mouche, P., Simon, B.: On the measure of the spectrum for the almost Mathieu operator. Commun. Math. Phys. 132, 103-118 (1990)

4. Bellissard, J., Lima, R., Testard, D.: A metal-insulator transition for the almost Mathieu model. Commun. Math. Phys. 88, 207-234 (1983)

5. Bellissard, J., Simon, B.: Cantor spectrum for the almost Mathieu equation. J. Funct. Anal. 48, 408-419 (1982)

6. Chambers, W.: Linear network model for magnetic breakdown in two dimensions. Phys. Rev. A 140, 135-143 (1965)

7. Choi, M.D., Elliott, G.A., Yui, N.: Gauss polynomials and the rotation algebra. Invent. Math. 99, 225-246 (1990)

8. Chulaevsky, V., Delyon, F.: Purely absolutely continuous spectrum for almost Mathieu operators. J. Stat. Phys. 55, 1279-1284 (1989)

9. Cycon, H.L., Froese, R.G., Kirsch, W., Simon, B.: Schrödinger operators. Berlin, Heidelberg, New York: Springer 1987

10. Delyon, F.: Absence of localization for the almost Mathieu equation. J. Phys. A 20, L21-L23 (1987)

11. Harper, P.G.: Single band motion of conduction electrons in a uniform magnetic field. Proc. Phys. Soc. Lond. A 68, 874-892 (1955)

12. Helffer, B., Sjostrand, J.: Semi-classical analysis for Harper's equation. III. Cantor structure of the spectrum. Mém. Soc. Math. France (N.S.) 39, 1-139 (1989)

13. Hofstadter, D.R.: Energy levels and wave functions of Bloch electrons in a rational or irrational magnetic field. Phys. Rev. B 14, 2239-2249 (1976)

14. Falconer, K.J.: The geometry of fractal sets. Cambridge: Cambridge University Press 1985

15. Fröhlich, J., Spencer, T., Wittwer, P.: Localization for a class of one dimensional quasi-periodic Schrödinger operators. Commun. Math. Phys. 132, 5-25 (1990) 
16. Hardy, G.H., Wright, E.M.: An introduction to the theory of numbers, Fifth ed. Oxford: Oxford University Press 1979

17. Last, Y.: A relation between a.c. spectrum of ergodic Jacobi matrices and the spectra of periodic approximants. Commun. Math. Phys. 151, 183-192 (1993)

18. Last, Y., Wilkinson, M.: A sum rule for the dispersion relations of the rational Harper's equation. J. Phys. A 25, 6123-6133 (1992)

19. Simon, B.: Almost periodic Schrödinger operators: a review. Adv. Appl. Math. 3, 463-490 (1982)

20. Sinai, Ya.G.: Anderson localization for one-dimensional difference Schrödinger operator with quasiperiodic potential. J. Stat. Phys. 46, 861-909 (1987)

21. Thouless, D.J.: Bandwidth for a quasiperiodic tight binding model. Phys. Rev. B 28, 4272-4276 (1983)

22. Thouless, D.J.: Scaling for the discrete Mathieu equation. Commun. Math. Phys. 127, 187-193 (1990)

23. Thouless, D.J., Tan, Y.: Total bandwidth for the Harper equation. III. Corrections to scaling. J. Phys. A 24, 4055-4066 (1991)

24. Thouless, D.J., Tan, Y.: Scaling, localization and bandwidths for equations with competing periods. Physica A 177, 567-577 (1991)

25. Toda, M.: Theory of nonlinear lattices, 2nd Ed., Chap. 4. Berlin, Heidelberg, New York: Springer 1989

26. Watson, G.I.: WKB analysis of energy band structure of modulated systems. J. Phys. A 24, 4999-5010 (1991)

Communicated by T. Spencer 